\title{
A survey on local binary pattern and gabor filter as texture descriptors of smart profiling systems
}

\author{
Shihab Hamad Khaleefah ${ }^{1}$, Salama A. Mostafa ${ }^{2}$, Aida Mustapha ${ }^{3}$, Noor Azah Samsudin ${ }^{4}$, \\ Mohammad Faidzul Nasrudin ${ }^{5}$, Abdullah Baz ${ }^{6}$ \\ ${ }^{1,2,3,4}$ Faculty of Computer Science and Information Technology, Universiti Tun Hussein Onn Malaysia, Malaysia \\ ${ }^{5}$ Centre for Artificial Intelligence Technology, Universiti Kebangsaan Malaysia, Malaysia \\ ${ }^{6}$ Department of Computer Engineering, College of Computer and Information Systems, \\ Umm Al-Qura University, Saudi Arabia
}

\begin{tabular}{l} 
Article Info \\
\hline Article history: \\
Received Apr 1, 2020 \\
Revised Jun 11, 2020 \\
Accepted Jun 28, 2020 \\
\hline Keywords: \\
Feature extraction \\
Gabor filter \\
Image processing \\
Local binary pattern \\
Texture descriptors
\end{tabular}

\begin{abstract}
With the dramatic expansion of image information nowadays, image processing and computer visions are playing a significant role in terms of several applications such as image classification, image segmentation, pattern recognition, and image retrieval. One of the important features that have been used in many image applications is texture. The texture is the characteristic of a set of pixels that formed the image. Therefore, analyzing such texture would have a significant impact on segmenting the image or detecting important portions of such image. This paper aims to overview the feature extraction and description techniques depicted in the literature to characterize regions for images. In particular, two of popular descriptors will be examined including Local Binary Pattern (LBP) and Gabor Filter. The key characteristic behind such investigation lies in how the features of an image would contribute toward the process of recognition and image classification. In this regard, an extensive discussion is provided on both LBP and Gabor descriptors along with the efforts that have been intended to combine them. The reason behind investigating these descriptors is that they are considered the most common local and global descriptors used in the literature. The overall aim of this survey is to show current trends on using, modifying and adapting these descriptors in the domain of image processing.
\end{abstract}

Copyright $\odot 2020$ Institute of Advanced Engineering and Science. All rights reserved.

\section{Corresponding Author:}

Shihab Hamad Khaleefah, Salama A. Mostafa, Aida Mustapha,

Faculty of Computer Science and Information Technology,

Universiti Tun Hussein Onn Malaysia, Malaysia.

Email:shi90hab@gmail.com

\section{INTRODUCTION}

In the area of image processing and computer vision, the texture indicates the duplication of basic texture elements called texels. Such element consists of multiple pixels that are either intended to be randomly placed or in a periodic manner. According to [1] an image texture would be coarse, fine, smooth, granulated, rippled, regular, irregular or linear. Generally speaking, the texture reflects a neighbour surrounding point the same way that colour would reflect a point value [2]. In this vein, the scale would be a significant factor that is associated with the texture in which a variant scale would lead to variant textures even if the textures were equivalent [3]. Therefore, it would make a sense if a single image would contain multiple levels of different textures located in different scales.

In fact, there are several methods that have been used in the literature regarding the texture analysis. However, [4] have provided a taxonomy for such strategies inside four main categories as well as Statistical, Structural, Model-based, and Signal Processing as shown in Figure 1. The statistical methods aim to handle 
the task of texture analysis as a statistical task in which the frequency of specific positions of pixels can be examined. The structural methods aim to handle the texture analysis using structural properties such as the texture primitives or so-called texels. Such analysis aims to identify the structure of these texels and their relationship. The third class which is the model-based methods have been intended to examine the construction of an image in order to analyze the texture by capturing the perceived qualities of the image texture. The fourth class which is the signal processing methods aim at assigning a filtering approach in order to filter the frequency of texture description. The following sections will discuss the latter-mentioned methods in detail.

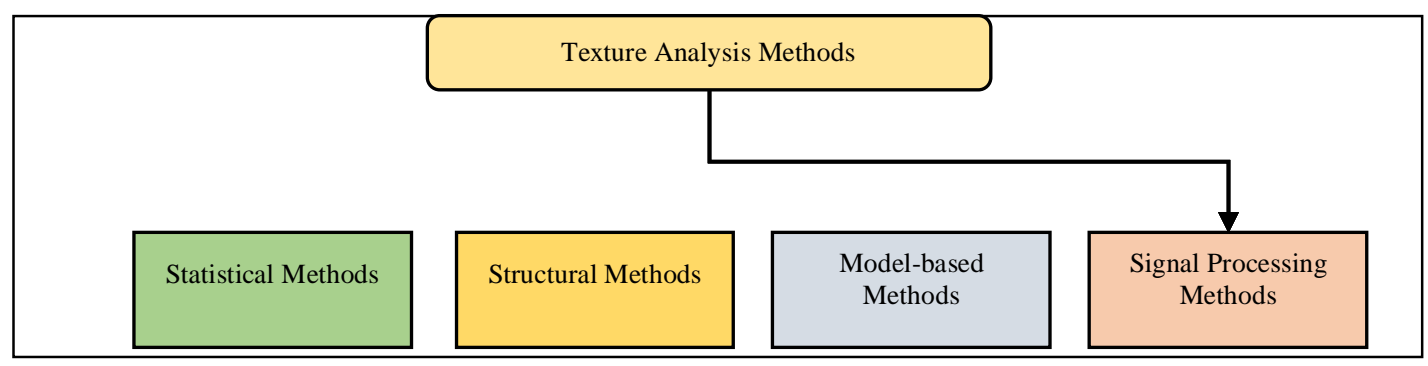

Figure 1. Taxonomy of texture analysis methods

The paper is organized as; Section 2 provides a brief background of texture descriptors, Section 3 concentrates on the Local Binary Pattern (LBP) and its current trends of using such a technique. Section 4 concentrates on the Gabor filter in which the mechanism of this technique and its current trend usage is discussed. Finally, Section 5 highlights the research efforts that attempted to combine both descriptors.

\section{TEXTURE DESCRIPTORS}

This section aims to tackle the methods that have been used to describe the texture. Following sub-sections will discuss each method independently.

\subsection{Statistical Methods}

The statistical method is one of the earliest methods that have been used to describe an image texture. It aims to analyze the qualities of texture using the spatial distribution of the intensity value. This can be performed by computing the variance of the pixel value from the gray-level histogram of an image. One of the common statistical descriptors was the (GLCM) which has been introduced by Haralick and Shanmugam [5]. Such matrix provides the statistical information of an image related to its distribution of pixels pairs. However, since the co-occurrence matrix has some drawbacks such as the challenging task of tuning the parameters, Baraldi and Parmiggiani [4] have proposed the gray-level difference statistics where the features are computed by examination the intensity values with the combination of intensity values or the typical intensity.

\subsection{Structural (Geometrical) Methods}

Structural elements of an image which are called texels or textons. Such elements are the smallest portions that articulate the textured surface. Generally speaking, the structural descriptors are relatively constant in terms of the illuminations however, it relies on the determination of texels. Bela Julesz [6] have claimed that the psychophysical aspect would significantly contribute toward the structural descriptors in which the human eye has the ability to differentiate the texture with different texels.

\subsection{Model-based Methods}

Such methods aim at determining model parameters by analyzing a specific texture and then utilize it as features. One of the common approach used by these methods is the Markov Random Fields (MRF) which aims to analyze the contextual information of an image. The hypothesis behind such an approach lies in the fact that the intensity of every pixel relies on the intensity of neighbouring pixels [7]. In this vein, MRF will treat the pixels as a series of state probabilities in which the state of a pixel is being defined by the states of its surrounding pixels. 


\subsection{Signal-Processing (Filtering) Methods}

Generally, the human brain has the ability to analyze the frequency information of intensities which has a significant impact on the textures [8]. Signal processing methods aim at performing filtering approach on the image then records the responses as texture features. Such filters are being used to examine both time and frequency or so-called spatial domain filters [9]. In this manner, the spatial filters attempt to provide the information related to edge density per unit are to extract the information as unique texture features. The Fourier transform is applied in spatial domain analysis on the image in order to extract the texture features. One of the popular filters that have been used in the literature was the Gabor filter which has been proposed for the purpose of signal representation in which a function is being used to utilize both time and frequency. Hence, this paper will focus on two methods including statistical and signal processing in which the Local Binary Pattern which is a statistical method, and the Gabor Filter which is a signal processing method are being discussed in detail.

\section{LOCAL BINARY PATTERN}

LBP has been introduced by Ojala et al. [10] which has been demonstrated a powerful grayscale invariant texture descriptor in the literature. LBP operator integrates both characteristics of structural and statistical texture analysis. LBP aims to illustrate the texture using micro-primitives and their statistical placement rules. In fact, the LBP performs on a pixel basis, and at the same time, illustrating the eight surrounding pixels in binary code. After that, it summarizes all codes into a histogram which facilitate the extraction of texture feature. This means that 256 texture patterns for the $3 \times 3$ neighbouring would be produced. Given a matrix as follow:

$$
B=\left(\begin{array}{ccc}
g_{8} & g_{1} & g_{2} \\
g_{7} & g(0,0) & g_{2} \\
g_{6} & g_{5} & g_{4}
\end{array}\right)
$$

The above matrix shows a $3 \times 3$ grayscale block of pixels in which the centre at the location $(0,0)$. In this manner, LBP will subtract the coordinate from each neighbour as follow:

$$
L B P 1=\left(\begin{array}{ccc}
g_{8}-g_{\text {center }} & g_{1}-g_{\text {center }} & g_{2}-g_{\text {center }} \\
g_{7}-g_{\text {center }} & g_{\text {center }} & g_{3}-g_{\text {center }} \\
g_{6}-g_{\text {center }} & g_{5}-g_{\text {center }} & g_{4}-g_{\text {center }}
\end{array}\right)
$$

Now, in order to generate the binary code, the (3) should be considered:

$$
\begin{aligned}
& L B P 2=\left(\begin{array}{ccc}
s\left(g_{8}-g_{\text {center }}\right) & s\left(g_{1}-g_{\text {center }}\right) & s\left(g_{2}-g_{\text {center }}\right) \\
s\left(g_{7}-g_{\text {center }}\right) & g_{\text {center }} & s\left(g_{3}-g_{\text {center }}\right) \\
s\left(g_{6}-g_{\text {center }}\right) & s\left(g_{5}-g_{\text {center }}\right) & s\left(g_{4}-g_{\text {center }}\right)
\end{array}\right) \\
& s(x)= \begin{cases}1 & \text { if } x \geq 0 \\
0 & \text { if } x<0\end{cases}
\end{aligned}
$$

Consequentially, the eight-bit binary pattern will be encoded as:

$$
L B P=\sum_{p=0}^{8} s\left(g_{p}-g_{c}\right) 2^{p}
$$

The 256 possible patterns that would be produced by (3) will be used to construct the histogram which will facilitate the process of texture description.

\subsection{Current Trends of Using LBP}

Local Binary Pattern is one of the effective texture feature analysis methods which has been examined for several domains in the literature. For instance, Liu et al. [11] have addressed the problem of sensitivity of LBP regarding the noisy data which hinders the capturing of macro-structure information. The authors have proposed a novel texture classification method based on a median robust extended LBP. Unlike the traditional ones, the proposed method constrains the regional image medians instead of the raw image intensities. Using benchmark data, the authors have demonstrated that their proposed method has a superior performance regarding the grayscale variations and noise-resistance. On the other hand, Wan et al. [12] have proposed an enhanced LBP namely Average-LBP for the process of texture analysis on 
human breast tissue images. Basically, such images are taken by the Optical Coherence Microscopy (OCM) which is a technology to capture microscopic images of human tissue. The proposed averaged-LBP has outperformed the original one in terms of encoding the texture structure.

With the availability of real-time 3D sensors such as Kinect, the efforts of gesture recognition have extensively progressed. Since the 2-d LBP utilizes the texture information thus, it cannot be applied for gesture recognition which usually does not have texture information. Therefore, Kim et al. [13] have proposed an adaptive LBP for 3-d hand tracking. The proposed method has the ability to be invariant to both rotation and the depth distance in range images. Similarly, Dey et al. [14] have addressed the problem of word spotting in which the digital libraries are usually storing their books as images. Therefore, there is a vital demand to process these images in order to detect the words. This would offer a great opportunity for information retrieval where the user can search using some keywords and get accurate results. Hence, the authors have proposed a combination of LBP and spatial sampling for the process of detecting or spotting handwritten words using large-scale historical documents. Comparing the results of texture analysis features with other methods, the proposed method has demonstrated better performance.

Additionally, Almezoghy et al. [15] have proposed a method for human palmprint detection using LBP. The proposed method utilizes different morphological features, then the LBP will use such feature in order to perform the detection. Using real data collected from 500 people, the proposed method has been applied and compared with other methods such as Principle Component Analysis. Experimental results showed that the proposed method has superior performance compared to PCA. Bian et al. [16] have proposed an extension of LBP which is called multi-structure LBP for the process of classifying high-resolution images. The proposed method utilizes three coupled descriptors with multi-structure sampling in order to identify complementary features. Results revealed that the proposed method has the ability to capture local spatial pattern and local contrast effectively compared to other methods.

Jia et al. [17] have improved the classification of hyperspectral images using a novel LBP namely LBP superpixel-level. The proposed method utilizes a uniform LBP to identify local features. Consequentially, a Support Vector Machine classifier has been used to classify the description of every pixel belonging to every class. Using real data of hyperspectral images, the proposed method showed superior performance in terms of classification.

Due to the limitation of traditional LBP in capturing spatial structures, Yuan et al. [18] have proposed a Hamming-Distance approach to the LBP for texture classification and material recognition. The experiments demonstrate the efficacy of the proposed method. Another study by Xu et al. [19] have addressed the problem of averaging an underlying smooth surface in an image when using the original LBP. Therefore, the authors have proposed a Polynomial Contrast Binary Pattern (PCBP) in order to efficiently estimate the underlying local surface information which can be depicted as a linear projection of the local patch. The authors have examined the proposed method for the facial recognition task. Finally, Kou et al. [20] have examined the limitation of the inability to capture the macro and microstructure of an image when using the traditional LBP. The authors have proposed a Principal Curvatures Local Binary Pattern (PCLBP). The proposed method had the ability to capture consecutive rotation invariance which led to improving the extraction of micro and macrostructure texture information. Table 1 summarizes all the LBP texture analysis related work that has been illustrated in latter paragraphs.

Table 1. Summary of the current trend on LBP

\begin{tabular}{cccc}
\hline Reference & Method & Problem & Task \\
\hline Liu et al. [11] & extended LBP & Structure & Image Classification \\
Wan et al. [12] & Average-LBP & Averaging & Medical image classification \\
Kim et al. [13] & adaptive LBP & Structure & Image classification for handwritten recognition \\
Dey et al. [14] & LBP and spatial sampling & Structure & Image segmentation for handwritten recognition \\
Almezoghy et al. [15] & PCA-LBP & Averaging & Image Classification for Palm recognition \\
Bian et al. [16] & multi-structure LBP & Structure & High-resolution image classification \\
Jia et al. [17] & LBP superpixel-level & Structure & Image Classification for hyperspectral images \\
Yuan et al. [18] & HDLBP for spatial structure & Structure & Image classification for material recognition \\
Xu et al. [19] & PCBP & Averaging & Face recognition \\
Kou et al. [20] & PCLBP & Structure & Image texture Classification \\
\hline
\end{tabular}

As shown in both Table 1 and Figure 2, two main limitations existed in the original LBP tackled by the recent studies including averaging and structure. However, the studies that examined the structure were larger than the averaging. This demonstrates the demand for modifying the original LBP to suite some spatial structure problems. 


\section{CURRENT TREND ON LBP}

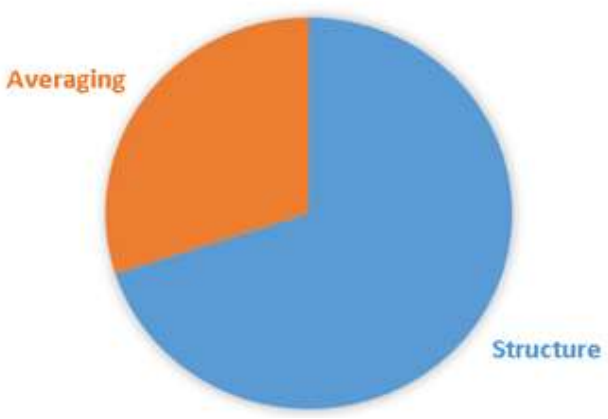

Figure 2. Main limitations of LBP tackled by recent studies

\section{GABOR FILTER AS A SIGNAL PROCESSING METHOD}

Gabor filter is composed of the product among an elliptical Gaussian and a sinusoidal. In fact, both Gaussian and sinusoidal have the same rotation that can be represented by x-axis and y-axis. 1-D Gabor elementary function can be defined as the product of a pulse of a form of a probability function with a harmonic oscillation of any frequency:

$$
g(t)=e^{-\alpha^{2}(t-t 0)^{2}} e^{i 2 \pi f_{0} t+\emptyset}
$$

where $\alpha$ is the time duration and bandwidth of the Gaussian envelope, t0 indicates the centroid, f0 indicates the frequency of sinusoidal and $\varnothing$ indicates the phase shift. In this vein, the 1-d Fourier transform of the Gabor function depicted in (5) can be represented as follow:

$$
G(f)=\sqrt{\frac{\pi}{\alpha^{2}}} e^{-\left(\frac{\pi}{\alpha}\right)^{2}(f-f 0)^{2}} e^{-i 2 \pi(f-f 0)+\emptyset}
$$

The Gabor function in (6) was focused near the time instance t0. Assume adjusting both t0 and $\emptyset$ to zero, the Gabor function will be represented as:

$$
g(t)=e^{-\alpha^{2} t^{2}} e^{i 2 \pi f_{0} t}
$$

\subsection{2-D Gabor Filter}

The two dimensional Gabor filter has been proposed for the image processing in general and for the feature extraction and texture analysis in particular. In the two dimensions Gabor, the time $t$ is being replaced with spatial coordinates (i.e. $\mathrm{x}$-axis and $\mathrm{y}$-axis). As well as, the frequency $\mathrm{f}$ is being also replaced with two other variables which are $\mathrm{u}$ and $\mathrm{v}$. In this vein, 2-D Gabor function can be represented as follow:

$$
g(x, y)=g(x, y ; f 0, \theta)=e^{-\left(\alpha^{2} x_{p}^{2} B^{2} y_{p}^{2}\right)} e^{i 2 \pi f_{0} x_{p}}(8)
$$

where $x_{p}=\mathrm{x} \cos \theta+\mathrm{y} \sin \theta, y_{p}=-\mathrm{x} \sin \theta+\mathrm{y} \cos \theta$, and $\theta$ is the rotation angle of the Gaussian major axis and the plane wave (sinusoidal). Apparently, a 2-D Gabor filter is the product of a 2-D Gaussian with oriented sinusoids.

\subsection{Current Trends on Using Gabor Filter}

Gabor filter has been widely used for several domains especially the medical domain. For example, Lu et al. [21] have addressed the problem of low-quality images of the fundus in the medical domain. The authors have utilized a combination of Gabor filter and matched filter in order to recognize the vessels textures within the fundus images. Experimental results showed that the combination of Gabor and matched filters have significantly improved the quality of the vessel. In addition, Radhakrishnan et al. [22] have addressed another medical topic which is skin diseases. In fact, there are many skin diseases that can be detected in early stages such as tumour and skin cancer. Therefore, the authors have designed and implemented a new Gabor algorithm for the process of detecting skin diseases. The new algorithm has been called a Variable Thresholding Gabor Algorithm. During the image segmentation of skin diseases, there are 
several parameters and variables that should be tuned. In the meantime, tuning these variables is different in accordance with the variety of skin diseases. Therefore, the proposed variable Thresholding algorithm will set a threshold value during the decision making period in order to overcome this problem.

On the other hand, the Gabor filter has been used for geographical applications such as the one proposed by Ang et al. [23]. Basically, the authors have examined the problem of the footprint that still remain after disasters such as earthquakes. These footprints would be noise data that can hinder the process of detecting or predicting faults. Therefore, the authors have proposed a modified Gabor filter in order to detect and remove these footprints. Experimental results showed that the Gabor filter had the ability to detect and remove the footprint which has contributed toward better identification of structural features such as the sand and channels. Khaleefah et al. [24] have utilized texture classification method by using LBP descriptors for the task of paper texture identification or fingerprinting, which are well-known technique, in the texture classification that shown superior performance in authenticating documents. Tadic et al. [25] have proposed a fuzzy Gabor filter for the license plates detection. In fact, the authors have addressed the problem of selecting the parameters of the Gabor filter. Therefore, they have used the fuzzy theory on the wavelength of the Gabor filter. Such a fuzzy-based algorithm has contributed toward efficient extraction of features with little noisy data. Low et al. [26] have proposed a multi-fold Gabor filter Convolution (FGFC) which aims to select appropriate filter for the task of face recognition. This is due to the tremendous possibilities existed in using the traditional Gabor filter.

Khaleefah et al. [27] have used multiple Gabor filters for the task of an Automated Paper Fingerprinting (APF) method. The authors' work includes applying a number of Gabor filters with different scaling [S] and orientation [O]. $\mathrm{S}$ is $\{3,5,7,9,11\}$ and $\mathrm{O}$ is $\{0, \pi / 4, \pi / 2,3 \pi / 4, \pi\}$ prior to the LBP operators $\left(\mathrm{LBP}_{\mathrm{P}, \mathrm{R}}^{\mathrm{basic}}, \mathrm{LBP}_{\mathrm{P}, \mathrm{R}}^{\mathrm{u} 2}\right.$ and $\left.\mathrm{LBP}_{\mathrm{P}, \mathrm{R}}^{\text {riu2 }}\right)$ which results, $\left(\mathrm{GFLBP}_{\mathrm{P}, \mathrm{R}}^{\mathrm{basic}}, \mathrm{GFLBP}_{\mathrm{P}, \mathrm{R}}^{\mathrm{u} 2}\right.$ and $\left.G F \mathrm{LBP}_{\mathrm{P}, \mathrm{R}}^{\mathrm{riu} 2}\right)$ operators. Experiments showed that the proposed method is able to identify deformed papers fingerprinting. Similarly, in the field of face recognition, there are variations of the selection for Gabor filter bank which leads to many experiments adjustment (trial and error). Hence, there are complex computations required to identify the best practice. Therefore, Dora et al. [28] have proposed a meta-heuristic approach of Particle Swarm Optimization (PSO) with the Gabor filter in order to identify the best parameters and minimizing the required computations. Ji et al. [29] have proposed a combination of Gabor filter and Discrete Fourier Transform in order to improve the multi-scale structures which cannot be detected by using Gabor filter only. Babashakoori and Ezoji [30] have proposed a combination of Gabor filter and Hough transform in order to improve the diameter measurement in scanning electron microscopy images. Table 2 summarizes all the Gabor filter related work that has been illustrated in the latter paragraphs.

Table 2. Summary of the current trend on gabor filter

\begin{tabular}{cccc}
\hline Reference & Method & Problem & Task \\
\hline Khaleefah et al. [27] & $\begin{array}{c}\text { Multi-scale Gabor filter, Multi-orientation } \\
\text { Gabor filter and Multi-fold Gabor filter }\end{array}$ & Parameter tuning & Texture document image \\
Lu et al.[21] & Gabor and matched filters & Low quality & Medical image segmentation \\
Radhakrishnan et al. [22] & Variable thresholding Gabor algorithm & Parameter tuning & Medical image classification \\
Ang et al. [23] & Modified Gabor filter & Noisy data & Geographical image classification \\
Khaleefah et al. [24] & Gabor filter & Structure & Texture document image \\
Tadic et al. [25] & Fuzzy-based Gabor filter & Parameter tuning & Pattern recognition \\
Low et al. [26] & FGFC & Parameter tuning & Face recognition \\
Dora et al. [28] & PSO with Gabor filter & Parameter tuning & Face Recognition \\
Ji et al. [29] & Gabor with DFT & Structure & Image classification \\
Babashakoori and Ezoji [30] & Gabor and Hough & Structure & Image classification \\
\hline
\end{tabular}

As shown in Table 2 and Figure 3, the most frequent limitation that still exists in the use of Gabor filter is the parameter tuning where tremendous possibilities can be selected. Therefore, many research efforts have attempted to transform the problem into optimization where the aim is to find the best solution. 


\section{CURRENT TREND ON GABOR FILTER}

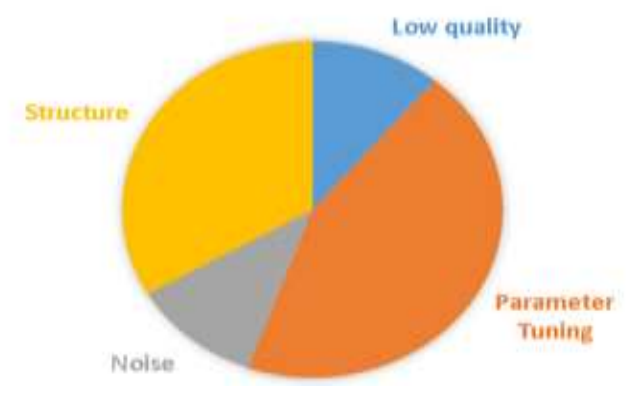

Figure 3. Main limitations of gabor filter tackled by recent studies

\section{COMBINED TEXTURE DESCRIPTORS}

Various studies have addressed the combination of LBP and Gabor filter, for example, Zhou et al. [31] have proposed multiple descriptors including LBP, Local Phase Quantization (LPQ) and Gabor filter in order to solve the problem of blur or low-resolution of face representation. Experiments showed that the combination has significantly overcome the blur situation when representing faces. Hadizadeh [32] has proposed a combination of Gabor filter along with LBP in order to improve the texture classification. Both descriptors have been used to extract local and global features. Experiments showed that the proposed combination has improved the classification accuracy, especially when encountering Gaussian noise. Tao et al. [33] have proposed a combination of multi-channel local features produced by LBP along with Gabor filter for face recognition. Experiments demonstrated the efficacy of the proposed combination, especially for gray-level differences. Huang et al. [34] have combined both LBP and Gabor filter for object recognition from video. The proposed LBP has been intended to extract the local features, while the Gabor filter has been employed to extract global features. Consequentially, the Principle Component Analysis (PCA) has been applied in order to reduce the dimensionality of the features. The classification has been performed using Support Vector Machine (SVM) and showed that the combination of such descriptors has improved the accuracy of detection.

For the sake of extending local and global features, Liu et al. [35] have proposed a combination of 2-D Gabor filter along with LBP for facial expression recognition. The proposed combination has been incorporated with an Extreme Learning Machine (ELM) in order to perform the classification. Shihab et al., [36] study the phenomenon of Automated Paper Fingerprinting (APF) method that deploys a combination approach for Gabor Filters (GF) and Uniform Local Binary Patterns (ULBP) called the GFULBP operator to cater for both local and global image information during the feature extraction process for higher texture classification accuracy. The authors further reported that the APF method is evaluated by a standard dataset of 306 blank paper images derived from pre-existing scanner image dataset from the different institution (Universiti Kebangsaan Malaysia (UKM)) with properties ranges from 50 DPI, 100 DPI, and 150 DPI respectively. It was observed in their report that the images are captured by a flatbed scanner with 50 DPI, 100 DPI, and 150 DPI resolutions and each image is represented by four patches that are segmented from specific locations of the image. In addition the test results of the APF revealed that GFULBP is able to outperform the ULBP alone by $30.68 \%$ when the GF has a 5 scale and $\pi / 2$ orientation degree. Furthermore, this research indicates that the integration of Gabor filters and ULBP significantly enhances the feature extraction quality and fingerprinting accuracy. Table 3 summarizes the latter studies that addressed the combination of Gabor and LBP.

As shown in Figure 4, most of the studies that have examined the combination of Gabor and LBP were intended to extract more local and global features in order to overcome the specific issue.

Table 3. Summary of combined gabor and LBP studies

\begin{tabular}{cccc}
\hline Reference & Method & Problem & Task \\
\hline Zhou et al. [31] & Gabor + LBP + LPQ & Blur (low-resolution) & Face recognition \\
Hadizadeh [32] & Gabor + LBP & Extending local and global features & Texture classification \\
Tao et al. [33] & Gabor + LBP & Extending local and global features & Face recognition \\
Huang et al. [34] & Gabor + LBP + PCA & Extending local and global features & Object recognition \\
Liu et al. [35] & 2D Gabor + LBP & Extending local and global features & Face recognition \\
Khaleefah et al. [36] & Gabor + LBP & Parameter tuning & Paper fingerprinting \\
Alharan et al. [37] & Gabor + LBP & Extending local and global features & Texture classification \\
\hline
\end{tabular}




\section{COMBINATION OF GABOR AND LBP}

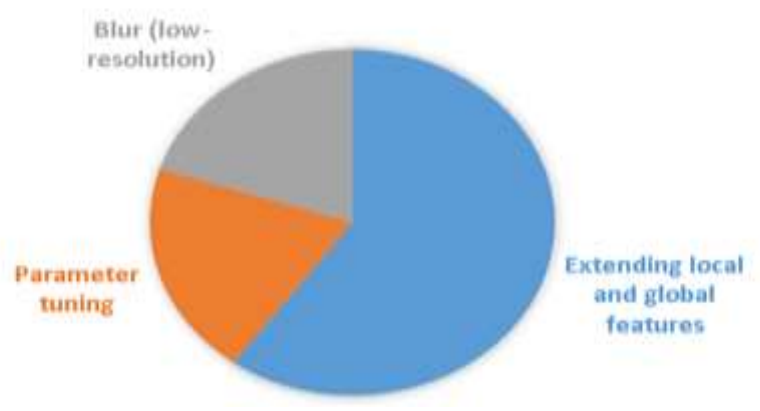

Figure 4. Studies combining gabor and LBP

\section{DISCUSSION}

Reviewing the literature of LBP and Gabor, one could notice that the most existing limitation behind these descriptors is the addressing the spatial structure and the parameter tuning respectively. Therefore, when analyzing the studies that combined both descriptors, it worth mentioning that the combination has contributed toward overcoming the aforementioned limitations. This has been represented where the global features produced by Gabor have facilitated to handling spatial structure. In contrast, the local features produced by the LBP has facilitated toward reducing the problem of parameter tuning in Gabor. Apart from the two main limitations, it was noticed that the combination has also contributed toward solving specific issues such as blur (low-resolution), Gaussian noisy and gray-level differences.

\section{CONCLUSION}

This paper provided an overview of the feature extraction and description techniques depicted in the literature to characterize regions for images. A background of texture analysis and texture descriptors have been given. In addition, more concentration has been done on both LBP and Gabor texture descriptors. From the literature, one could notice that the most widely used descriptors from the local side were the LBP, and from the global side was the Gabor filter. Finally, promising efforts have been discussed in regard to combining both local and global descriptors. This combination can improve the detection of a wide range of patterns and extraction accuracy. Such suggestions might facilitate future researches to be aware of every pros and con of each descriptor. The future work shall consider applying different combinations of LBP and Gabor filters in complex feature extraction modelling.

\section{ACKNOWLEDGEMENTS}

This study is supported by Multidisciplinary Research Grant, Vot H511, Universiti Tun Hussein Onn Malaysia and Universiti Kebangsaan Malaysia (UKM) under Research Grant PP-FTSM-2020.

\section{REFERENCES}

[1] S. Annadurai, "Fundamentals of digital image processing," Pearson Education India, 2007.

[2] S. Belongie, et al., "Color-and texture-based image segmentation using EM and its application to content-based image retrieval," pp. 675-682.

[3] D. C. Lee and T. Schenk, "Image segmentation from texture measurement," International Archives of Photogrammetry and Remote Sensing, vol. 29, pp. 195-195, 1993.

[4] A. Baraldi and F. Parmiggiani, "An investigation of the textural characteristics associated with gray level cooccurrence matrix statistical parameters," IEEE Transactions on Geoscience and Remote Sensing, vol. 33, no. 2, pp. 293-304, 1995.

[5] R. M. Haralick and K. Shanmugam, "Textural features for image classification," IEEE Transactions on systems, man, and cybernetics, no. 6, pp. 610-621, 1973.

[6] B. Julesz, "Textons, the elements of texture perception, and their interactions," Nature, vol. 290, no. 5802, pp. 9197, 1981.

[7] F. S. Cohen and D. B. Cooper, "Simple parallel hierarchical and relaxation algorithms for segmenting noncausal Markovian random fields," IEEE Transactions on Pattern Analysis and Machine Intelligence, no. 2, pp. 195-219, 1987. 
[8] M. D. Abràmoff, et al., "Image processing with ImageJ," Biophotonics international, vol. 11, no. 7, pp. 36-42, 2004.

[9] N. Kingsbury, "Complex wavelets for shift invariant analysis and filtering of signals," Applied and computational harmonic analysis, vol. 10, no. 3, pp. 234-253, 2001.

[10] T. Ojala, et al., "A comparative study of texture measures with classification based on featured distributions," Pattern recognition, vol. 29, no. 1, pp. 51-59, 1996.

[11] L. Liu, et al., "Median Robust Extended Local Binary Pattern for Texture Classification," IEEE Transactions on Image Processing, vol. 25, no. 3, pp. 1368-1381, 2016.

[12] S. Wan, et al., "Integrated local binary pattern texture features for classification of breast tissue imaged by optical coherence microscopy," Medical image analysis, vol. 38, pp. 104-116, 2017.

[13] J. Kim, et al., "An adaptive local binary pattern for 3d hand tracking," Pattern Recognition, vol. 61, pp. 139-152, 2017.

[14] S. Dey, et al., "Local Binary Pattern for Word Spotting in Handwritten Historical Document," Structural, Syntactic, and Statistical Pattern Recognition: Joint IAPR International Workshop, S+SSPR 2016, Mérida, Mexico, November 29 - December 2, 2016, Proceedings, in A. Robles-Kelly, et al. (eds), Cham, Springer International Publishing, pp. 574-583, 2016.

[15] H. H. Almezoghy, et al., "Human Identification with Palm Print Based on Local Binary Pattern," 2017.

[16] X. Bian, et al., "Extended multi-structure local binary pattern for high-resolution image scene classification," pp. 5134-5137.

[17] S. Jia, et al., "Local Binary Pattern-Based Hyperspectral Image Classification With Superpixel Guidance," IEEE Transactions on Geoscience and Remote Sensing, 2017.

[18] F. Yuan, et al., "Mixed co-occurrence of local binary patterns and Hamming-distance-based local binary patterns," Information Sciences, vol. 460-461, pp. 202-222, 2018.

[19] Z. Xu, et al., "Local polynomial contrast binary patterns for face recognition," Neurocomputing, vol. 355, pp. 1-12, 2019.

[20] Q. Kou, et al., "Principal curvatures based local binary pattern for rotation invariant texture classification," Optik, vol. 193, p. 162999, 2019.

[21] C. Y. Lu, et al., "Vessel enhancement of low quality fundus image using mathematical morphology and combination of Gabor and matched filter," pp. 168-173.

[22] K. Radhakrishnan, et al., "Design and implementation of high speed Gabor filter with variable thresholding process for disease detection," pp. 430-435.

[23] C. Ang, et al., "Residual Footprint Removal Using Gabor Filter."

[24] S. H. Khaleefah, et al., "Fingerprinting of deformed paper images acquired by scanners," in 2015 IEEE Student Conference on Research and Development (SCOReD), pp. 393-397, 2015.

[25] V. Tadic, et al., "Fuzzified Gabor filter for license plate detection," Engineering Applications of Artificial Intelligence, vol. 48, pp. 40-58, 2016.

[26] C. Y. Low, et al., "Multi-fold Gabor filter convolution descriptor for face recognition,” pp. 2094-2098.

[27] S. H. Khaleefah and M. F. Nasrudin, "Identification of printing paper based on texture using gabor filters and local binary patterns," Journal of Theoretical \& Applied Information Technology, vol. 86, no. 2, 2016.

[28] L. Dora, et al., "An evolutionary single Gabor kernel based filter approach to face recognition," Engineering Applications of Artificial Intelligence, vol. 62, pp. 286-301, 2017.

[29] H. Ji, et al., "Digital Gabor filters do generate MRA-based wavelet tight frames," Applied and Computational Harmonic Analysis, vol. 47, no. 1, pp. 87-108, 2019.

[30] S. Babashakoori and M. Ezoji, "Average fiber diameter measurement in Scanning Electron Microscopy images based on Gabor filtering and Hough transform," Measurement, vol. 141, pp. 364-370, 2019.

[31] S. R. Zhou, et al., "Local binary pattern (LBP) and local phase quantization (LBQ) based on Gabor filter for face representation," Neurocomputing, vol. 116, pp. 260-264, 2013.

[32] H. Hadizadeh, "Multi-resolution local Gabor wavelets binary patterns for gray-scale texture description," Pattern Recognition Letters, vol. 65, pp. 163-169, 2015.

[33] G. Tao, et al., "A novel texture description by local multi-channels Gabor comprehensive patterns," Optik International Journal for Light and Electron Optics, vol. 126, no. 19, pp. 2214-2218, 2015.

[34] W. Huang, et al., "Identification of group-housed pigs based on Gabor and Local Binary Pattern features," Biosystems Engineering, vol. 166, pp. 90-100, 2018.

[35] Z. T. Liu, et al., "Combining 2D Gabor and Local Binary Pattern for Facial Expression Recognition Using Extreme Learning Machine," Journal of Advanced Computational Intelligence and Intelligent Informatics, vol. 23, no. 3, pp. 444-455, 2019.

[36] S. H. Khaleefah, et al., "The ideal effect of Gabor filters and Uniform Local Binary Pattern combinations on deformed scanned paper images," Journal of King Saud University - Computer and Information Sciences, 2019.

[37] A. F. H. Alharan, et al., "A cluster-based feature selection method for image texture classification," Indonesian Journal of Electrical Engineering and Computer Science, vol. 14, no. 3, pp. 1433-1442, 2019. 